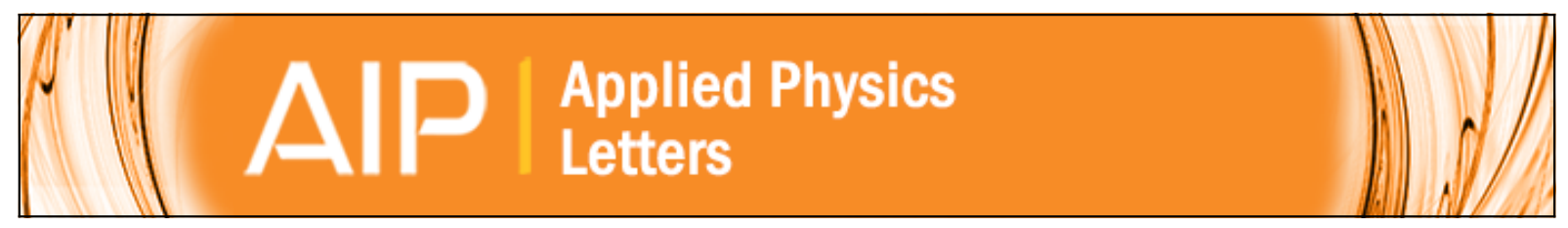

\title{
Nanoindentation-induced deformation of Ge
}

J. E. Bradby, J. S. Williams, J. Wong-Leung, M. V. Swain, and P. Munroe

Citation: Applied Physics Letters 80, 2651 (2002); doi: 10.1063/1.1469660

View online: http://dx.doi.org/10.1063/1.1469660

View Table of Contents: http://scitation.aip.org/content/aip/journal/apl/80/15?ver=pdfcov

Published by the AIP Publishing

\section{Articles you may be interested in}

Nanoindentation-induced phase transformation in relaxed and unrelaxed ion-implanted amorphous germanium J. Appl. Phys. 106, 093509 (2009); 10.1063/1.3255999

Giant pop-ins and amorphization in germanium during indentation

J. Appl. Phys. 101, 043524 (2007); 10.1063/1.2490563

Nanoindentation-induced deformation in Al-Pd-Mn single quasicrystals

Appl. Phys. Lett. 88, 073103 (2006); 10.1063/1.2178412

Evidence for nanoindentation-induced phase transformations in germanium

Appl. Phys. Lett. 86, 131907 (2005); 10.1063/1.1894588

Indentation-induced crystallization and phase transformation of amorphous germanium

J. Appl. Phys. 96, 1464 (2004); 10.1063/1.1766414

\section{AlP $\left.\right|_{\text {APL Photonics }}$}

APL Photonics is pleased to announce Benjamin Eggleton as its Editor-in-Chief

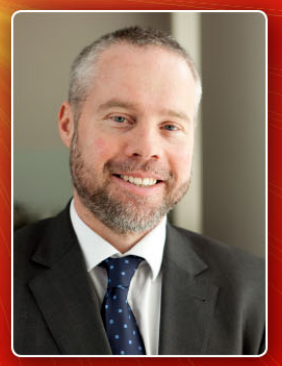




\title{
Nanoindentation-induced deformation of Ge
}

\author{
J. E. Bradby, ${ }^{\text {a) }}$ J. S. Williams, and J. Wong-Leung \\ Department of Electronic Materials Engineering, Research School of Physical Sciences and Engineering, \\ The Australian National University, Canberra, ACT 0200, Australia \\ M. V. Swain \\ Biomaterials Science Research Unit, Department of Mechanical and Mechatronic Engineering and Faculty \\ of Dentistry, The University of Sydney, Eveleigh, NSW 1430, Australia \\ P. Munroe \\ Electron Microscope Unit, University of New South Wales, Sydney, NSW 2052, Australia
}

(Received 15 November 2001; accepted for publication 15 February 2002)

\begin{abstract}
The deformation mechanisms of crystalline (100) Ge were studied using nanoindentation, cross sectional transmission electron microscopy (XTEM) and Raman microspectroscopy. For a wide range of indentation conditions using both spherical and pointed indenters, multiple discontinuities were found in the force-displacement curves on loading, but no discontinuities were found on unloading. Raman microspectroscopy, measured from samples which had plastically deformed on loading, showed a spectrum shift from that in pristine Ge, suggesting only residual strain. No evidence (such as extra Raman bands) was found to suggest that any pressure-induced phase transformations had occurred, despite the fact that the material had undergone severe plastic deformation. Selected area diffraction pattern studies of the mechanically damaged regions also confirmed the absence of additional phases. Moreover, XTEM showed that, at low loads, plastic deformation occurs by twinning and dislocation motion. This indicates that the hardness of $\mathrm{Ge}$ measured by indentation is not primarily dominated by phase transformation, rather by the nucleation and propagation of twin bands and/or dislocations. (C) 2002 American Institute of Physics. [DOI: 10.1063/1.1469660]
\end{abstract}

The mechanical properties and deformation mechanisms of semiconductors are important due to the considerable technological significance of these materials. It has been known for the more than a decade from diamond anvil experiments that the normal diamond-cubic phase of Si (Si-I) undergoes phase transformations to a range of higher density phases (Si-II, Si-III, and/or Si-XII) under hydrostatic pressure application and release. ${ }^{1,2}$ Similarities in the structure between $\mathrm{Si}$ and Ge suggest that Ge may also undergo densification during hydrostatic loading. ${ }^{3,4}$ Indeed, studies have shown that the high pressure phase transformations of Ge are broadly analogous to those in $\mathrm{Si}$, with $\mathrm{Ge}-\mathrm{I}$ reported to transform to a metallic $\beta$-Sn phase, Ge-II, under hydrostatic loading at $\sim 10.6 \mathrm{GPa},{ }^{3}$ and to further transform on pressure release from Ge-II to Ge-III and/or Ge-IV depending on the rate of pressure release. ${ }^{3}$

Although diamond anvil experiments are crucial in the deformation behavior of bulk materials under pure hydrostatic pressure, the behavior of semiconductors under nanoindentation is of more relevance to the semiconductor industry. Under indentation, with significant components of shear stress, it is conceptually not clear whether semiconductors should primarily deform via phase transformation or slip. However, of relevance to the indentation behavior of semiconductors is a proposal by Gilman ${ }^{5}$ that the prime mode of plastic deformation, and hence the hardness should be controlled by the onset of phase transformation. Indeed, this prediction has been verified in indentation studies of $\mathrm{Si}$

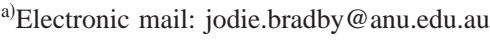

at room temperature, where discontinuities in both the load ("pop-in") and unloading ("pop-out") sections of the forcedisplacement curves, indicative of plastic deformation, have been correlated with phase transformations induced under the indenter. ${ }^{6-9}$ Hence, although slip can be observed in indented $\mathrm{Si}^{9}{ }^{9}$ the prime deformation process, which determines hardness during room temperature indentation, is phase transformation. Indentation has been used to study the mechanical deformation of $\mathrm{Ge}$, but in considerably less detail than $\mathrm{Si}$. To date, it is not possible to establish whether the major deformation process in Ge under indentation is phase transformation or slip. For example, although discontinuities on loading (pop-in) have been widely reported in Ge by a number of authors, ${ }^{10-13}$ no discontinuity on unloading (popout) has been reported. There is some evidence $e^{6,10,12-16}$ that Ge can undergo an indentation-induced phase transformation during high-load, pointed indentation, but other studies ${ }^{11}$ that suggest that $\mathrm{Ge}$ can deform without phase transformation. In cases where phase transformations were observed in Ge, there has been little attempt to correlate such changes with discontinuities in the load-unload curves. Hence, it is not yet possible to establish whether the onset of plastic deformation in Ge at room temperature, which determines hardness, is controlled by a phase transformation or other processes. In this study, we investigate the mechanical deformation mechanisms induced by low load spherical and pointed indentation of $\mathrm{Ge}$ to determine the mode of deformation during the onset of plasticity.

A series of indentations were made in crystalline (100) $\mathrm{Ge}$ at loads of up to $250 \mathrm{mN}$. The indentation device used 

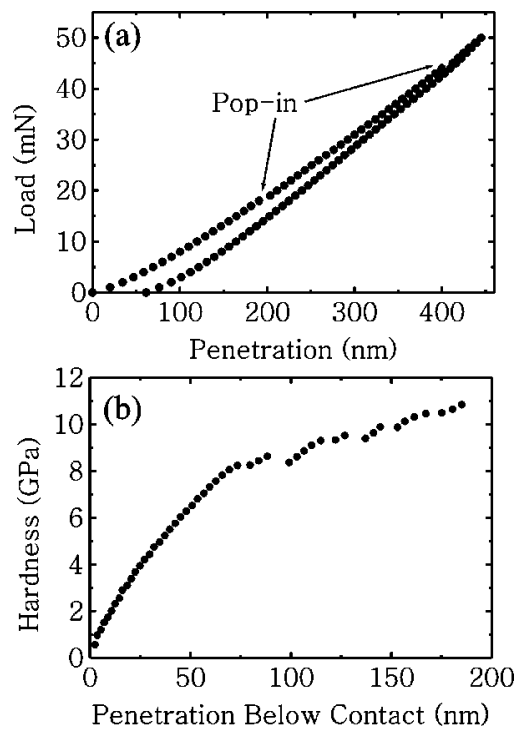

FIG. 1. (a) Load-unload curve of Ge to a maximum load of $50 \mathrm{mN}$ with a spherical indenter of radius $\sim 4.2 \mu \mathrm{m}$. (b) Hardness versus penetration below contact for Ge.

was the Ultra-micro Indentation System-2000 (UMIS) with either a spherical indenter of $\sim 4.2 \mu \mathrm{m}$ radius or a Berkovich indenter at ambient pressure and room temperature. Raman spectra were collected using a Renishaw 2000 Raman imaging microscope with the $632.8 \mathrm{~nm}$ excitation lines of a helium-neon laser and a spot size of $\sim 1-2 \mu \mathrm{m}$. Any laserinduced phase changes were avoided by keeping the laser intensity low. Cross sectional transmission electron microscopy (XTEM) samples were prepared from indents using an FEI $\times$ P200 focused ion beam (FIB) with Ga ions at $30 \mathrm{keV}$. To protect the surface of the samples during the ion milling process a $\sim 1 \mu \mathrm{m}$ thick layer of platinum was deposited over the surface of the indents using the FIB instrument. Ge was found to be especially sensitive to ion-beam-induced damage so ion currents were kept low $(<70 \mathrm{pA})$ when imaging the surface. The transmission electron microscope used in this study was a Philips CM 300 operated at an accelerating voltage of $300 \mathrm{kV}$.

Typical of the load-unload behavior for Ge with both spherical and pointed indenters in this study, discontinuities (pop-in events) during loading with a spherical indenter can be seen (denoted with arrows) in the curve shown in Fig. 1(a). It is interesting to note that plastic deformation is characterized by multiple pop-in events for Ge. Such a deformation process has been previously observed in materials where slip (not phase transformation) is the main mechanism of deformation. ${ }^{17}$ Also characteristic of indentation data from $\mathrm{Ge}$, is that no discontinuities (pop-out events) were observed on unloading. The hardness as a function of indenter penetration below contact, measured by the partial load-unload method of Field and Swain, ${ }^{18}$ is shown in Fig. 1(b). There is no strong evidence of the 'overloading' behavior that has been observed in some other semiconductor materials. ${ }^{8}$ Instead, Fig. 1(b) shows a series of small discontinuities (corresponding to pop-in events), with the first occurring at $\sim 8$ $\mathrm{GPa}$. The value of the hardness continues to increase gradually after each pop-in, suggesting appreciable work hardening.

Figure 2 shows a Raman spectrum from a spherical in-

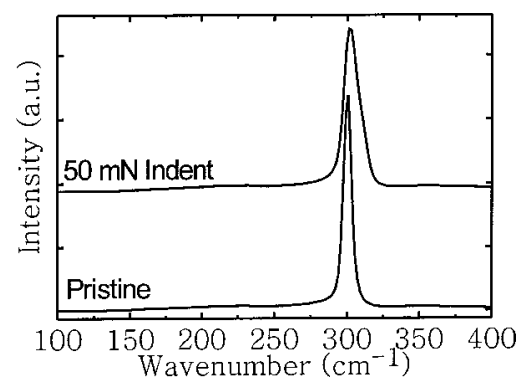

FIG. 2. Raman spectra from pristine Ge and a spherical indent loaded to a maximum load of $50 \mathrm{mN}$ at a loading and unloading rate of $\sim 7 \mathrm{mN} / \mathrm{s}$.

dent with a maximum load of $50 \mathrm{mN}$ compared with a spectrum taken from a pristine region of the sample. The Raman spectrum from pristine $\mathrm{Ge}$ has a band at $300 \mathrm{~cm}^{-1}$ corresponding to the diamond cubic Ge-I structure. The spectrum taken from the residual indent impression shows a small shift in the Raman band to $303 \mathrm{~cm}^{-1}$. Gogotsi et al. ${ }^{11}$ reported similar shifts in the Raman spectra taken from pointed indents with a maximum load of $70 \mathrm{mN}$ and attributed these small shifts to residual compressive stress within the indentations. No extra bands, expected if a phase transformed material has occurred, were found in the spectrum from the indent as compared to that of the pristine material. This again strongly suggests that the plastic deformation mechanism in our case is not by phase transformation.

A $[\overline{4} 00]$ two-beam bright-field XTEM image of a spherical indent at a maximum load of $50 \mathrm{mN}$ is shown in Fig. 3(a). This is typical of many XTEM samples we have studied in Ge after plastic deformation using both spherical and pointed indenters. A dense array of deformation, containing some twins and dislocation tangles, can be seen just below the surface. A second defect array is visible extending from $\sim 0.6 \mu \mathrm{m}$ to $\sim 1.3 \mu \mathrm{m}$ from the surface. These two defects arrays are joined by long twin bands which extend up to $\sim 1.4 \mu \mathrm{m}$ into the material. No cracking was observed at this

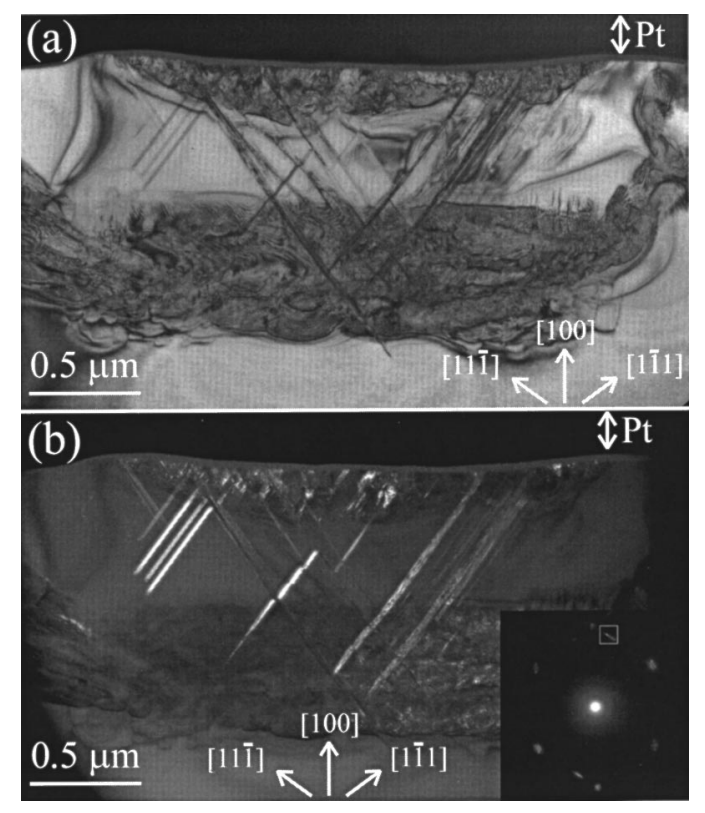

FIG. 3. XTEM images of a spherical indent in Ge at a maximum load of 50 $\mathrm{mN}$ and a loading and unloading rate of $\sim 7 \mathrm{mN} / \mathrm{s}$. (a) Bright-field [ $\overline{4} 00]$ two-beam image. (b) DF image taken using twin reflection (boxed) in the inset. Inset shows diffraction pattern taken from deformed region. 
maximum load $(50 \mathrm{mN})$. Selected area diffraction patterns (SADP) of the mechanically damaged regions were carefully studied at a number of orientations and showed no evidence of phase transformed material. The inset in Fig. 3(b) shows a SADP with extra streaky spots evidence of twin formation in this material (see boxed reflection). A dark-field (DF) image of the same region at the same magnification as Fig. 3(a) is shown in Fig. 3(b) using the highlighted twin reflection. Clearly highlighted in the DF image are twin bands parallel

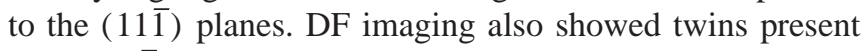
on the (1 $1 \overline{1} 1)$ planes. A closer inspection shows displacement of slip bands close to the intersection with other bands. This twinning and slip behavior is consistent with shear stress introduced along the $\{111\}$ planes during indentation. Note that slip along the remaining two $\{111\}$ planes $[(\overline{1} \overline{1} 1)$ and $(\overline{1} 1 \overline{1})]$ can not be clearly imaged in this geometry. The second deeper deformation array is clearly the region where the twins and/or dislocation slip bands intersect in the foil. Thus, despite the fact that only selected slip bands can be imaged in the geometry shown in Fig. 3, when they intersect and are pinned, a range of other (observable) defects appear to be formed to relieve stress at this depth.

The above experimental data clearly indicates that the onset of plastic deformation in $\mathrm{Ge}$, and hence the measured hardness, is determined by twinning and/or dislocation generation and not by phase transformation. The XTEM image shows a dense array of near-surface deformation under the indenter and this region contains some twin bands. On subsequent loading, some twin bands propagate more deeply into the material, where in some cases they appear to intersect and interact. These interactions may well impede the process of twinning/slip and this behavior may be the explanation for the work hardening effects observed in the hardness data shown in Fig. 1(b). For example, pinning of twin/ slip bands may result in an increase in pressure to initiate and propagate subsequent further bands. Consequently, this work hardening process may lead, at high maximum loads (and possibly at high loading rates), to a situation where densification by phase transformation may be more energetically favorable than plastic deformation by further twinning or slip. Hence, we propose the following deformation scenario. The initial plastic response of Ge under indentation is to deform by twinning and/or dislocation propagation (slip). On further severe loading, a phase transformation may be subsequently initiated if near-surface work hardening occurs.

Contrary to Gilman's prediction that the hardness of Ge is controlled by phase transformations, ${ }^{5}$ we note that no phase transformation was observed under the loading conditions presented here. This may be due, in part, to the different ratios of shear and hydrostatic stress components beneath spherical and pointed indenters. Indeed, evidence of phase transformations from previous studies of the indentation loading of $\mathrm{Ge}$ comes from indentation with pointed indenters, under either cyclical loading or with high maximum loads. ${ }^{6,12-15}$ Interestingly, high resolution scanning electron microscopy of indents in Ge made with a Berkovich indenter at a load of $50 \mathrm{mN}$ have reported slip steps and large amounts of extruded material. ${ }^{10,16}$ However, a recent report by Gogotsi et al. ${ }^{11}$ suggested that Ge displayed both transformation and dislocation motion and, depending on the loading conditions, one or the other can dominate. In particular, Raman spectra taken from low load $(70 \mathrm{mN})$ pointed indentations showed no evidence of any phase transformation. ${ }^{11}$ Yet, indents formed at higher loads, ${ }^{14}$ showed extra Raman bands, reported to correspond to the metastable high-pressure and amorphous phases of Ge. These results are in agreement with the deformation scenario we are proposing for Ge in which the material first undergoes plastic deformation via twinning and slip but then may undergo a phase transformation at higher loads if the twinning/ slip processes are subsequently impeded.

In conclusion, we have found that the indentationinduced deformation of $\mathrm{Ge}$, under the loading conditions used in this study, is predominantly by twinning aligned along the $\{111\}$ planes. Thus, unlike $\mathrm{Si}$, it appears that the hardness of $\mathrm{Ge}$ at room temperature, as measured by indentation, is not dominated by the initiation of a phase transformation. Other reports of more severe loading of Ge suggest that phase transformations can occur under more severe loading and we explain this behavior in terms of work hardening by pinning of slip bands, whereby a phase transformation may become energetically favorable. Additional TEM and Raman microspectroscopy (ideally in situ) studies at a range of loading conditions and temperatures seem to be desirable in order to better understand the subsequent phase transformation process under severe loading.

The authors would like to acknowledge Dr. V. OtienoAlego at the University of Canberra, Australia for assistance with Raman microspectroscopy. J.W.-L. is grateful to the Australian Research Council for funding under the fellowship program.

${ }^{1}$ J. Z. Hu, L. D. Merkle, C. S. Menoni, and I. L. Spain, Phys. Rev. B 34, 4679 (1986)

${ }^{2}$ R. O. Piltz, J. R. Maclean, S. J. Ackland, P. D. Hatton, and J. Crain, Phys. Rev. B 52, 4072 (1995).

${ }^{3}$ C. S. Menoni, J. Z. Hu, and I. L. Spain, Phys. Rev. B 34, 362 (1986).

${ }^{4}$ R. J. Nelmes, M. I. McMahon, N. G. Wright, D. R. Allan, and J. S. Loveday, Phys. Rev. B 48, 9883 (1993).

${ }^{5}$ J. J. Gilman, J. Mater. Res. 7, 535 (1992).

${ }^{6}$ D. R. Clarke, M. C. Kroll, P. D. Kirchner, R. F. Cook, and B. J. Hockey, Phys. Rev. Lett. 60, 2156 (1988).

${ }^{7}$ T. Page, W. C. Oliver, and C. J. McHargue, J. Mater. Res. 7, 450 (1992).

${ }^{8}$ J. S. Williams, Y. Chen, J. Wong-Leung, A. Kerr, and M. V. Swain, J. Mater. Res. 14, 2338 (1999).

${ }^{9}$ J. E. Bradby, J. S. Williams, J. Wong-Leung, M. V. Swain, and P. Munroe, J. Mater. Res. 16, 1500 (2001).

${ }^{10}$ S. V. Hainsworth, A. J. Whithead, and T. F. Page, in Plastic Deformation of Ceramics, edited by R. C. Bradt, C. A. Brookes, and J. L. Routbort (Plenum, New York, 1995), p. 173.

${ }^{11}$ Y. G. Gogotsi, V. Domnich, S. N. Dub, A. Kailer, and K. G. Nickel, J. Mater. Res. 15, 871 (2000).

${ }^{12}$ G. M. Pharr, W. C. Oliver, R. F. Cook, P. D. Kirchner, M. C. Kroll, T. R. Dinger, and D. R. Clarke, J. Mater. Res. 7, 961 (1992).

${ }^{13}$ S. N. Dub, J. Superhard. Mats. 21, 32 (1999).

${ }^{14}$ A. Kailer, K. G. Nickel, and Y. G. Gogotsi, J. Raman Spectrosc. 30, 939 (1999).

${ }^{15}$ S. J. Lloyd, J. M. Molina-Aldareguia, and W. J. Clegg, J. Mater. Res. 16, 3347 (2001).

${ }^{16}$ T. F. Page, L. Riester, and S. V. Hainsworth, Mater. Res. Soc. Symp. Proc. 522, 113 (1998).

${ }^{17}$ J. E. Bradby, S. O. Kucheyev, J. S. Williams, J. Wong-Leung, M. V. Swain, P. Munroe, G. Li, and M. R. Phillips, Appl. Phys. Lett. 80, 383 (2002).

${ }^{18}$ J. S. Field and M. V. Swain, J. Mater. Res 8, 297 (1993). 